\title{
Comparison of receptor affinity of natSc-DOTA-TATE
}

versus nat(Ta-IDOTA-TATE

Eftychia Koumarianou ${ }^{1}, 2$, Dariusz Pawlak ${ }^{1}$, Agnieszka Korsak, Renata Mikolajczak ${ }^{1}$

${ }^{1}$ National Centre for Nuclear Research, Radioisotope Centre POLATOM, Otwock, Poland

${ }^{2}$ Radiology Department, Duke University Medical Center, Durham, United States

[Received 20 X 2011; Accepted 16 XI 2011]

\section{Abstract}

BACKGROUND: ${ }^{44} \mathrm{Sc}$ as a positron emitter can be an interesting alternative to ${ }^{68} \mathrm{Ga}\left(\mathrm{T}_{1 / 2}=67.71 \mathrm{~min}\right)$ due to its longer half-life $\left(\mathrm{T}_{1 / 2}=3.97 \mathrm{~h}\right)$. Moreover, the $\beta^{-}$emitter ${ }^{47} \mathrm{Sc}$ can be used for therapy when attached to the same biomolecule vectors. DOTA as a chelating agent has been proven suitable for the radiolabelling of peptides recognising tumour cell receptors in vivo with $\mathrm{M}^{3+}$ radiometals. DOTA-derivatized peptides have been successfully labelled with ${ }^{90} \mathrm{Y}$ and ${ }^{177} \mathrm{Lu}$ for therapy, and with ${ }^{68} \mathrm{Ga}$ for PET imaging. However, published data on ${ }^{44} \mathrm{Sc}$-labelled DOTA-biomolecules as potential PET radiotracers are still very limited. The aim of this study was to com-

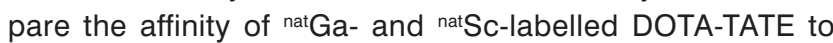
somatostatin receptors subtype 2 expressed in rat pancreatic cancer cell line AR42J.

MATERIAL AND METHODS: The cold complexes of DOTA-TATE with nat $G a$ and nat $\mathrm{Sc}$ were synthesized and identified by HPLC and MS analysis and evaluated in vitro for competitive binding to cancer cell line AR42J expressing somatostatin receptors subtype 2 (sstr2).

Correspondence to: Renata Mikolajczak, Ph.D

National Centre for Nuclear Research,

Radioisotope Centre POLATOM

05-400 Otwock. Poland

Tel: +48 2271807 01, fax:+48 2271803 50, PO Box: 3808 email: r.mikolajczak@polatom.pl
RESULTS: The IC50 values calculated from the displacement curve of [ $\left.{ }^{125} \mid-\mathrm{Tyr}^{11}\right]$-SST-14 were: $0.20 \pm 0.18,0.70 \pm 0.20,0.64$

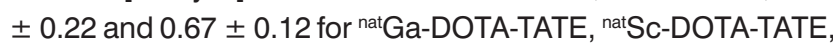
DOTA-TATE, and [Tyr $\left.{ }^{11}\right]$-SST-14 complexes, respectively, with the affinity lowering in the decreasing order: ${ }^{\text {natGa-DOTA-TATE }}$ $>$ DOTA-TATE $>$ Tyr ${ }^{11}$-SST-14 $>$ natSc-DOTA-TATE. CONCLUSIONS: The binding affinity of natGa-DOTA-TATE appeared higher than that of natSc-DOTA-TATE. Further in vitro and in vivo studies are needed to verify the influence of the chelated metal on the affinity and uptake of the respective radiolabelled compounds. This information might be crucial when the in vivo applications of peptides labelled with ${ }^{68} \mathrm{Ga}$ and ${ }^{44} \mathrm{Sc}$ for PET, as well as the use of ${ }^{47} \mathrm{Sc}$ for radiotherapy are considered.

Key words: scandium-44, gallium-68, PET tracers, receptor affinity, DOTA-derivatised peptides

Nuclear Med Rev 2011; 14, 2: 85-89

\section{Introduction}

In recent years peptide receptor radionuclide therapy (PRRT) has utilized synthetic peptides as vectors for radionuclides such as ${ }^{90} \mathrm{Y}$ and ${ }^{177} \mathrm{Lu}$. This has been accomplished with the aid of positron emission tomography (PET), which involves the same vector biomolecules labelled with positron emitters, and was first demonstrated with ${ }^{68} \mathrm{Ga}$-labelled somatostatin (SST) analogues for diagnostic imaging of neuroendocrine tumours [1]. Since then growing interest in other positron emitters obtained in generator systems has been observed [2, 3]. The increasing availability of new radionuclides with diagnostic and therapeutic properties offers new possibilities for individualized nuclear medicine options.

${ }^{44} \mathrm{Sc}$ is a positron emitter radionuclide $\left[\mathrm{E}_{\beta+} \max =1475.4 \mathrm{keV}\right.$, $\left.E_{r}=1157.0 \mathrm{keV}(99.9 \%)\right]$ with a half life of 3.97 hours, which can be utilized for diagnostics with ${ }^{47} \mathrm{Sc}$ as a matched pair for radiotherapy. Additionally, Grignon et al. [4] reported that ${ }^{44} \mathrm{Sc}$ is an interesting radionuclide for nuclear medicine imaging using $\beta^{+}-\gamma$ coincidences. The use of ${ }^{44} \mathrm{Sc}$ with a half-life more than 3 times longer than that of ${ }^{88} \mathrm{Ga}\left(\mathrm{T}_{1 / 2}=67.71 \mathrm{~min}\right)$ makes it an useful alternative for diagnostic purposes but also for dosimetry and 
further therapy planning with the use of biomolecules labelled with the $\beta^{-}$emitting ${ }^{47} \mathrm{Sc}$ as radiotherapeutic agents [5]. The availability of ${ }^{44} \mathrm{Sc}$ is also increasing $[3,6]$.

The chemistry of $\mathrm{Sc}^{3+}$ is similar to that of the lanthanides, and the "lanthanide like" elements. Due to its small ionic radius it is also chemically similar to aluminium and gallium [7]. The thermodynamic stability constant of the 1,4,7,10-teraazacyclododecane-N,N',N',N"'-teraacetic acid (DOTA) complex with $\mathrm{Ga}^{3+}$ is in the range from 21.3 to 26.1 [8, 9], with $\mathrm{Sc}^{3+}$ it is 27.0 [10], while the following values of 26.7, 23.9, 29.2, 25.95 have been reported for $\mathrm{Lu}^{3+}[7,8,11,12]$. All of the above values are similar as indicated from the data reported by Viola-Villegas and Doyle [13]. Hence chelators developed for the complexation of gallium, and the lanthanides can also be used for the complexation of scandium.

Generally, small neuropeptides, such as somatostatin (SST) and gastrin releasing peptide (GRP)/bombesin (BN) analogues, labelled with $\gamma$ - and/or $\beta$--emitting radionuclides are investigated for their ability to bind to receptors which are overexpressed in a variety of malignant tumours [14-16]. The affinity of the designed chelator-peptide construct to these receptors may vary depending on the metal incorporated into the complex [17]

The published results of the comparative in vitro and in vivo study of DOTA-BN[2-14] $\mathrm{NH}_{2}$ labelled with ${ }^{90} \mathrm{Y}$ and ${ }^{177} \mathrm{Lu}$ as well as with ${ }^{44} \mathrm{Sc}$ and ${ }^{68} \mathrm{Ga}$ revealed differences in the in vitro and in vivo behaviour of these complexes, which could be attributed to the influence of metal on the complex receptor affinity $[18,19]$. The present study is focused on the in vitro binding affinity of the $\mathrm{M}^{3+}$ type radiometals of Sc and Ga complexed with [DOTA, Tyr ${ }^{3}$, Thr ${ }^{8}$ ] octreotide (DOTA-TATE), which is a clinically used somatostatin analogue $[20,21]$.

\section{Material and methods}

\section{Chemicals}

DOTA-TATE was purchased from piChem (Austria). [ ${ }^{125}$-Tyr $\left.{ }^{11}\right]$ SST-14 and $\left[\right.$ Tyr $\left.^{11}\right]$-SST-14 were purchased from Perkin-Elmer Life and Analytical Sciences (USA). All other chemicals and materials were used as supplied and were of analytical grade unless otherwise stated.

Cold complexes of the peptide with natGa and natSc were synthesized and identified by high pressure liquid chromatography (HPLC) and mass spectrometry (MS).

\section{High pressure liquid chromatography (HPLC)}

The HPLC system for the quality control of the cold complexes was equipped with UV-VIS detector. The analysis was performed using a reverse phase C-18 Luna column (Phenomenex, USA). The mobile phase was a gradient of $0.1 \%$ TFA (Trifluoroacetic Acid) $/ \mathrm{H}_{2} \mathrm{O}$ (Solvent A) and 0.1\% TFA/Acetonitrile (ACN) (Solvent B). The elution scheme of solvent $B$ was $0 \%$ for 2 min, increased to $40 \%$ from 2 to $9 \mathrm{~min}$, remaining at $40 \%$ until $15 \mathrm{~min}$, and then decreased to $0 \%$ in $3 \mathrm{~min}$ from $18 \mathrm{~min}$ to 23 remaining at $0 \%$, at a flow rate of $0.6 \mathrm{ml} / \mathrm{min}$.

\section{Cold complexes of DOTA-TATE with nat Sc and nat $\mathrm{Ga}$}

The cold metal complexes of DOTA-TATE were synthesized and identified according to the previously described method [18]. Briefly, $100 \mu \mathrm{g}$ of DOTA-TATE was dissolved in $250 \mu \mathrm{l}$ ammonium acetate $0.4 \mathrm{M}, \mathrm{pH} 5$, or $250 \mu \mathrm{l}$ of ascorbic acid $(100 \mathrm{mg} / \mathrm{ml})$ was added. An appropriate amount of nat $\mathrm{ScCl}_{3}$ or nat $\mathrm{GaCl}_{3}$ solution ( $1 \mathrm{mg} / \mathrm{ml}$ in $0.05 \mathrm{M} \mathrm{HCl}$ ) was added to obtain a molar ratio of DOTA-TATE to metal 1:5. The sample was incubated at $95^{\circ} \mathrm{C}$ for 25 minutes and left to cool down to room temperature. The cold complexes were analyzed by HPLC. The samples were purified from free metal by Solid Phase Extraction (SPE) using pre-conditioned C-18 columns (100 mg resin, Sep-Pak, Waters), and the mobile phase consisted of $5 \mathrm{ml}$ of ethanol and $5 \mathrm{ml}$ of $0.9 \% \mathrm{NaCl}$. The samples were loaded on the cartridge followed by $5 \mathrm{ml} 0.9 \%$ $\mathrm{NaCl}$ (to elute non-bound natSc or nat $\mathrm{Ga}$ ) and by $3 \mathrm{ml}$ pure methanol (natSc-DOTA-TATE or natGa-DOTA-TATE fraction). The cold complex fractions were then lyophilized under vacuum giving a light yellow powder in both cases. The purified samples were also analyzed by Electron Spray lonization-Mass Spectrometry (ESI-MS).

\section{In vitro studies}

\section{Cell culture}

The rat pancreatic cancer cell line AR42J expressing somatostatin receptors subtype 2 (sstr2) was used for the in vitro experiments. The cell line was cultured in RPMI-1640 (Gibco Invitrogen) supplemented with $10 \%$ foetal calf serum (Gibco Invitrogen), antibiotics (streptomycin, $100 \mu \mathrm{g} / \mathrm{ml}$; penicillin, $100 \mathrm{U} / \mathrm{ml}$; Sigma Aldrich), and glutamax (Gibco Invitrogen). The cells were kept in a humidified atmosphere at $37^{\circ} \mathrm{C}$ in $5 \% \mathrm{CO}_{2}$. The cells were fed every 2 days and subcultured by trypsinization (0.05\% Trypsin-EDTA, Gibco Invitrogen) when the cells covered about $80 \%$ of the surface in the flask.

\section{Saturation curve of ${ }^{125 /-T y}{ }^{11}$-SST-14}

A saturation receptor assay for [125|-Tyr $\left.{ }^{11}\right]$-SST-14 was performed prior to the binding affinity studies in order to determine the minimum concentration required for the saturation of sstr. The cells were seeded in 24-well plates $\left(\sim 8 \times 10^{4}\right.$ cells/well) $48 \mathrm{~h}$ before the day of the experiment. On the day of the experiment the cells were incubated at $37^{\circ} \mathrm{C}$ in $5 \% \mathrm{CO}_{2}$ atmosphere for $90 \mathrm{~min}$ in the presence of increasing concentration of [125/-Tyr $\left.{ }^{11}\right]-S S T-14(0,20000,40000$, $60000,80000,100000$, and $120000 \mathrm{cpm}$, corresponding to $0,6.3$, $12.4,18.9,25.0,31.5$ and $37.8 \mathrm{pM}$, each in triplicate). At the completion of incubation the supernatant was collected and the cells were rinsed twice with $0.5 \mathrm{ml}$ of cold phosphate-buffered saline (PBS). The cells underwent lyses by addition of $1 \mathrm{~N} \mathrm{NaOH}$ and incubation at $37^{\circ} \mathrm{C} / 5 \% \mathrm{CO}_{2}$ for $10 \mathrm{~min}$. The radioactivity of the collected fractions was measured in order to determine the minimum required concentration. Experiments were performed in triplicate.

\section{Competitive binding studies}

The in vitro receptor binding affinity and specificity of Tyr ${ }^{11}$-SST-14, DOTA-TATE and its cold complexes with natSc and ${ }^{\text {nat } G a}$ in AR42J cells were determined by a competitive displacement cell-binding assay using the iodinated analogue [25I-Ty $\left.{ }^{11}\right]$-SST-14 according to the method previously described [18]. Briefly, $\sim 8 \times 10^{4}$ cells/well were seeded in 24 -well plates $48 \mathrm{~h}$ before the day of the experiment. On the day of the experiment the cells were incubated at $37^{\circ} \mathrm{C}$ in $5 \% \mathrm{CO}_{2}$ atmosphere for $1 \mathrm{~h}$ in the presence of $30,000-50,000 \mathrm{cpm}$ of ${ }^{225}$-[Tyr $\left.{ }^{11}\right]-S S T-14$ and 
increasing concentrations of the respective compound (from $1 \mathrm{pM}$ to $1 \mu \mathrm{M})$, each in triplicate. Upon completion of the incubation, the reaction medium was aspirated and the cells were washed twice with cold PBS. The cells underwent lyses by addition of $1 \mathrm{~N} \mathrm{NaOH}$ and incubation at $37^{\circ} \mathrm{C} / 5 \% \mathrm{CO}_{2}$ for $10 \mathrm{~min}$. The radioactivity of the collected fractions was measured in order to determine the $\mathrm{IC}_{50}$ value (inhibitory concentration, 50\%)

\section{Statistical methods}

The results were analyzed by non-linear regression analysis using GraphPad Prism (version TM, GraphPad software, San Diego California, USA).

\section{Results}

\section{Cold complexes of DOTA-TATE with natSc and nat $\mathrm{Ga}$}

The ESI-MS analysis of natGa-DOTA-TATE confirmed the presence of a single main complex at $764.2[\mathrm{~m} / \mathrm{z}]^{2+}$, which was in agreement with the calculated value (MW $=1505.3)$. The respective ESI-MS analysis of natSc-DOTA-TATE also confirmed the presence of a main peak at $761.5[\mathrm{~m} / \mathrm{z}]^{2+}$, being in agreement with the calculated value (MW =1498.5). Figures 1 and 2 present the mass spectrum and the HPLC profile of natSc-DOTA-TATE, respectively. For the determination of the exact concentration of the cold complexes used for the in vitro assays, the BCA Protein Assay was used (BCA kit, Thermo Scientific). A calibration curve of DOTA-TATE of GMP grade was used for the determination of the concentration of DOTA-TATE (R\&D grade), natSc-DOTA-TATE, and ${ }^{n a t} \mathrm{Ga}$-DOTA-TATE. The measured values were in good accordance with the calculated values

\section{Binding affinity studies}

AR42J cancer cells were incubated with increasing concentrations of [125|-Tyr $\left.{ }^{11}\right]$-SST-14 in order to measure specific radioligand binding at equilibrium so as to perform the displacement affinity study of the [Tyr $\left.{ }^{11}\right]-S S T-14$, DOTA-TATE, natSc-DOTA-TATE and,

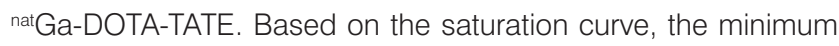

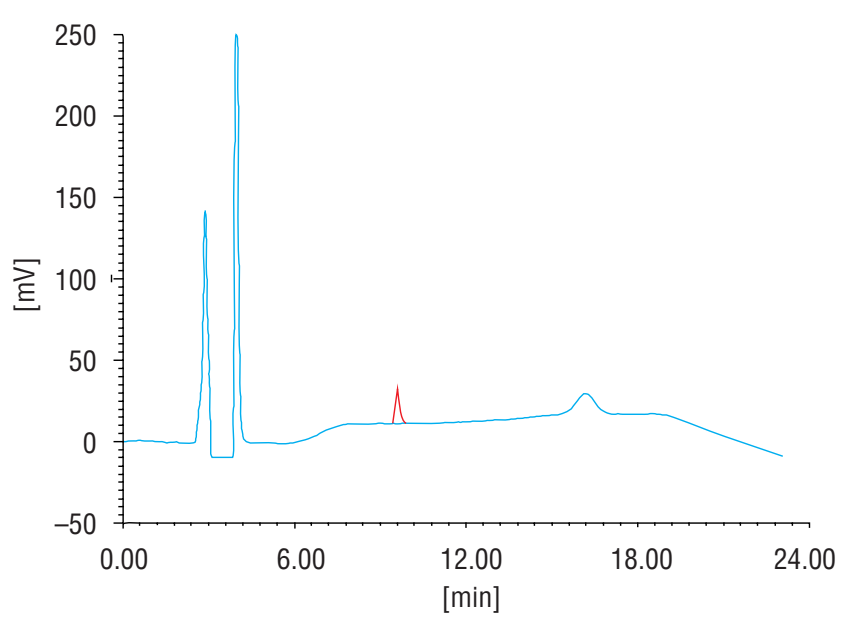

Figure 2. RP-HPLC analysis of natSc-DOTA-TATE (UV absorbance monitored at $220 \mathrm{~nm}$ ).

required amount of $\left[{ }^{125}-\mathrm{Tyr}^{11}\right]-\mathrm{SST} 14$ in order for the receptors to be saturated was in the range of 9.5-15.8 pM $(30,000-50,000$ $\mathrm{cpm})$, calculated according to the specific activity of the iodinated compound $(2200 \mathrm{Ci} / \mathrm{mmol})$.

The IC50 values calculated from the displacement curve of [ $\left.{ }^{125}{ }^{-T y}{ }^{11}\right]$-SST-14 were: $0.20 \pm 0.18,0.70 \pm 0.20,0.64 \pm 0.22$ and

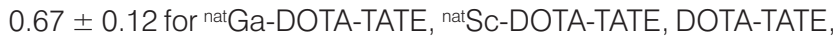
and [Tyr $\left.{ }^{11}\right]$-SST-14 complexes, respectively, as listed in Table 1. The values indicate the following affinity pattern: natGa-DOTA-TATE $>$ DOTA-TATE $>$ Tyr ${ }^{11}-$ SST-14 $>$ natSc-DOTA-TATE. The respective displacement curves are presented in Figure 3.

\section{Discussion}

Majkowska-Pilip and Bilewicz [10] evaluated the tri and tetraaza ligands for formation of macrocyclic complexes with Sc and compared them with analogous complexes of ${ }^{177} \mathrm{Lu}$ and ${ }^{68} \mathrm{Ga}$. The authors concluded that DOTA is the most suitable ligand for binding scandium radionuclides to biomolecules. Viola-Villegas and Doyle

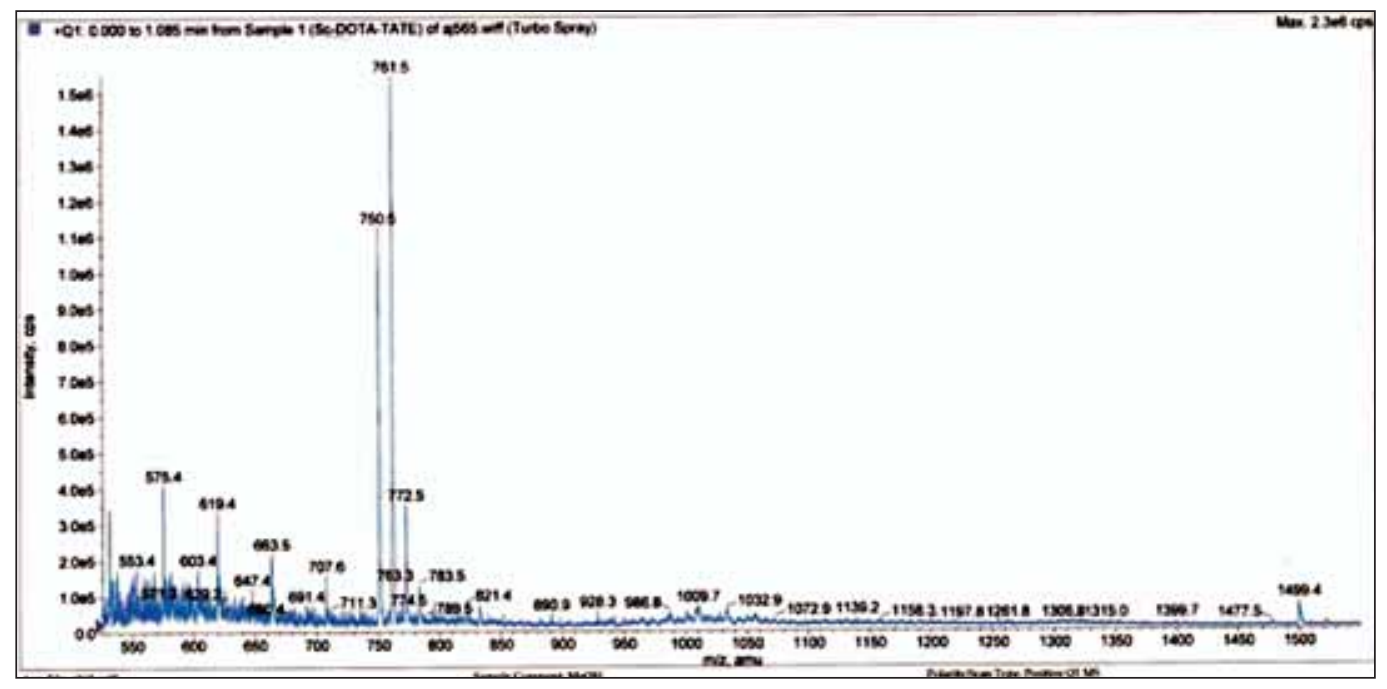

Figure 1. ESI-MS spectrum of natSc-DOTA-TATE. 


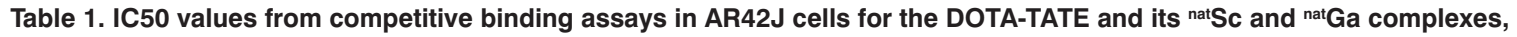
along with [Tyr11]-SST-14. The IC50 values of the complexes of the GRP analogue DOTA-BN[2-14]NH2 in PC-3 are given for comparison

\begin{tabular}{|c|c|c|c|}
\hline Derivative & $\begin{array}{c}\text { IC50 } \pm \text { SD }(\mathrm{nM}) \\
{\left[{ }^{125} \mid-\mathrm{Tyr}^{11}\right]-S S T-14} \\
\text { AR42J cells }\end{array}$ & Derivative & $\begin{array}{c}\text { IC50 } \pm \text { SD (nM) } \\
125 \mid-\left[\text { Tyr }^{4}\right]-B N \\
\text { PC3 cells }\end{array}$ \\
\hline$\left[\right.$ Tyr $\left.^{11}\right]$-SST-14 & $0.67 \pm 0.12$ & DOTA-BN[2-14] $\mathrm{NH}_{2}$ & $1.78 \pm 0.12^{*}$ \\
\hline DOTA-TATE & $0.64 \pm 0.22$ & natY-DOTA-BN[2-14] $\mathrm{NH}_{2}$ & $1.90 \pm 0.06^{*}$ \\
\hline natGa-DOTA-TATE & $0.20 \pm 0.18$ & natLu-DOTA-BN[2-14] $\mathrm{NH}_{2}$ & $1.34 \pm 0.11^{\star}$ \\
\hline \multirow[t]{2}{*}{ natSc-DOTA-TATE } & $0.70 \pm 0.20$ & natGa-DOTA-BN[2-14] $\mathrm{NH}_{2}$ & $0.85 \pm 0.06^{\star \star}$ \\
\hline & & natSc-DOTA-BN[2-14] $\mathrm{NH}_{2}$ & $6.49 \pm 0.13^{\star \star}$ \\
\hline
\end{tabular}

*Koumarianou et al. [18]; **Koumarianou et al. [19]

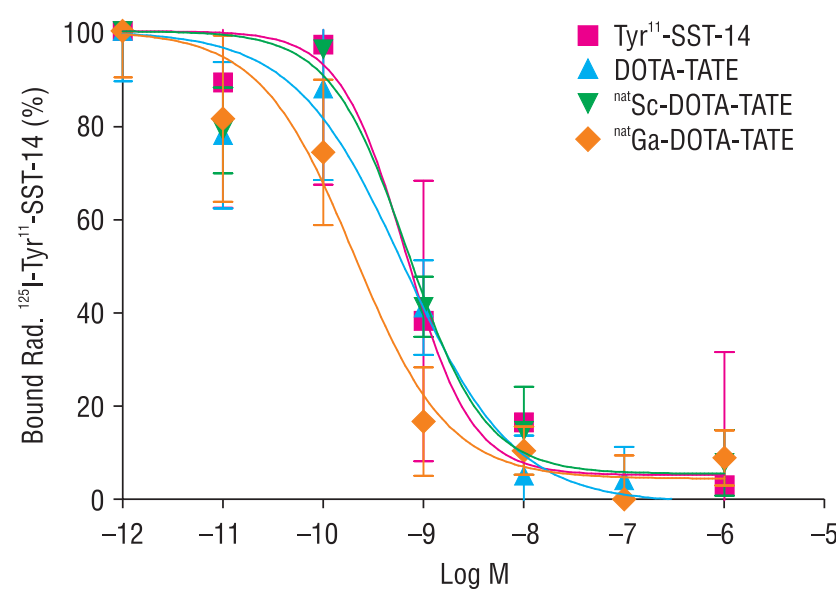

Figure 3. Displacement curves of [125I-Tyr $\left.{ }^{11}\right]-S S T-14$ from the competitive binding studies for Tyr ${ }^{11}$-SST-14, DOTA-TATE, natSc-DOTA-TATE, and natGa-DOTA-TATE

[13] indicated that there are differences in the crystal structure of DOTA with $\mathrm{Ga}$ and $\mathrm{Sc}$, suggesting at the same time a higher stability of Sc-DOTA complex. On the other hand, it has been shown previously by Reubi et al. [17] that DOTA can be a very efficient chelator for Y, Lu, and Ga when coupled to somatostatin analogues. The in vitro comparison indicated that not only the peptide sequence and conjugated chelator, but to a large extent also the metal involved in the complex formation influences the affinity of the molecule to the somatostatin receptors. Our previously published comparison of the in vitro and in vivo properties of another DOTA-conjugated peptide, ${ }^{90}$ Y-DOTA-BN[2-14] $\mathrm{NH}_{2}$, ${ }^{177}$ Lu-DOTA-BN[2-14] $\mathrm{NH}_{2}$, ${ }^{44} \mathrm{Sc}-\mathrm{DOTA}-\mathrm{BN}[2-14] \mathrm{NH}_{2}$, and ${ }^{68} \mathrm{Ga}-\mathrm{DOTA}-\mathrm{BN}[2-14] \mathrm{NH}_{2}$, revealed differences in the in vitro receptor affinity of these analogues (see Table 1) $[18,19]$. These differences may be attributed to the structural changes in the radioligand molecule, which influence the interaction with the receptor. The introduction of a certain metal or its replacement by another one may provoke considerable alterations in the in vivo binding affinity of a peptide to cell receptors and may have an important impact on the in vivo biodistribution of these radiopharmaceuticals.

So far, there is no published data on ${ }^{44}$ Sc-labelled peptides as PET tracer candidates in terms of in vitro and in vivo behaviour. Therefore, the main goal of this study was to evaluate the influence of the new radionuclide, ${ }^{44} \mathrm{Sc}$, on the receptor af- finity of another DOTA derivatized peptide which is already well established, such as the somatostatin analogue DOTA-TATE $[20,21]$. The ${ }^{68} \mathrm{Ga}-\mathrm{DOTA}$-TATE was used in direct comparison since ${ }^{68} \mathrm{Ga}$ complexes with DOTA chelated somatostatin analogues have been showing improved affinity to somatostatin receptor subtypes [17]. Considering the rather short half-life of ${ }^{68} \mathrm{Ga}$, the ${ }^{44} \mathrm{Sc}$ with $3.97 \mathrm{~h}$ half-life can be an interesting alternative for conjugation with biomolecules of longer metabolic half-life to allow late PET imaging.

\section{Summary}

In the present study natGa-DOTA-TATE showed slightly higher binding affinity to sst receptors of the AR42J cell line than natSc-DOTA-TATE, and the $\mathrm{IC}_{50}$ values of the studied derivatives were

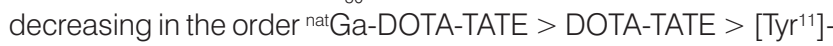
SST-14 $>$ natSc-DOTA-TATE. This relationship is in favour of Ga and is similar to the previously reported data for the DOTA-BN[2-14] $\mathrm{NH}_{2}$ derivatives $[18,19]$; however, the differences of affinity between Ga- and Sc-labelled DOTA-TATE are not so pronounced as they were in the case of DOTA-BN[2-14] $\mathrm{NH}_{2}$. Therefore, further studies are needed to verify if the influence of $\mathrm{Sc}$ radionuclides on the peptide affinity to certain receptors is critical for their diagnostic or therapeutic utility.

\section{Acknowledgements}

COST ACTION BM0607 Targeted Radionuclide therapy (TRNT), COST ACTION D38 Metal Based Systems for Molecular Imaging Applications and the Polish Ministry of Science and Higher Education grant No. 126/N-COST/2008/0.

\section{References}

1. Kowalski J, Henze M, Schuhmacher J, Mäcke HR, Hofmann M Haberkorn U. Evaluation of positron emission tomography imaging using $\left[{ }^{68} \mathrm{Ga}\right]-$ DOTA-D Phe(1)-Tyr(3)-Octreotide in comparison to $\left[{ }^{111} \mathrm{In}\right]$-DTPAOC SPECT. First results in patients with neuroendocrine tumors. Mol Imaging Biol 2003; 5: 42-48.

2. Welch MJ, and McCarthy TJ. The Potential Role of Generator-Produced Radiopharmaceuticals in Clinical PET. J Nucl Med 2000; 41: 315-317.

3. Rösch F, Knapp FF. Radionuclide generators. In: Vértes A, Nagy S, Klencsár Z, Rösch F (eds). Handbook of nuclear chemistry 2003; 4: 81-118, Kluver academic Publishers, the Netherlands. 
4. Grignon C, Barbet J, Bardies M et al. Nuclear medical imaging using $\beta^{+} \gamma$ coincidences from ${ }^{44} \mathrm{Sc}$ radio-nuclide with liquid xenon as detection medium. Nucl Instrum Methods Phys Res A 2007; 571: 142-145

5. Mausner LF, Joshi V, Kolsky KL et al. Evaluation of chelating agents for radioimmunotherapy with scandium-47. J Nucl Med 1995; 36: 104P.

6. Filosofov DV, Loktionova NS, Roesch F. A ${ }^{44} \mathrm{Ti} /{ }^{44} \mathrm{Sc}$ radionuclide generator for potential application of ${ }^{44} \mathrm{Sc}$-based PET-radiopharmaceuticals. Radiochim Acta 2010; 98: 149-156.

7. Pruszynski M, Loktionova NS, Filosofov DV, Roesch F. Processing of generator-produced ${ }^{44} \mathrm{Sc}$ for medical application — radiolabelling of DOTATOC with ${ }^{44}$ Sc. J Label Compd Radiopharm 2009; 52: S490.

8. NIST Standard Reference Database 46. Critically Selected Stability Constants of Metal Complexes Database. Compiled by: Smith RM Martell AE, Motekaitis RJ. Version 7.0 for Windows. 2003. US National Institute of Standards and Technology Standard Reference DATA Program; Gaithersburg, MD 20899

9. Polasek M, Kotek J, Hermann P, C sarova I, Binnemans K, Lukes I. Lanthanide(III) complexes of pyridine-N-oxide analogues of DOTA in solution and in the solid state. A new kind of isomerism in complexes of DOTA-like ligands. Inorg Chem 2009; 48: 466-475.

10. Majkowska-Pilip A, Bilewicz A. Macrocyclic complexes of scandium radionuclides as precursors for diagnostic and therapeutic radiopharmaceuticals. J Inorg Biochem 2011; 105: 313-320.

11. Loncin MF, Desreux JF, Merciny E. Coordination of lanthanides by two polyamino polycarboxylic macrocycles: formation of highly stable lanthanide complexes. Inorg Chem 1986; 25: 2646-2648.

12. Wu SL, Horrocks WD. Direct determination of stability constants of lanthanide ion chelates by laser-excited europium(III) luminescence spectroscopy: application to cyclic and acyclic aminocarboxylate complexes. J Chem Soc Dalton Trans 1997; 9: 1497-1502.
13. Viola-Villegas N, Doyle RP. The coordination chemistry of 1,4,7,10-tetraazacyclododecane-N,N',N"',N'"'-tetraacetic acid (H4DOTA): structural overview and analyses on structure-stability relationships. Coordination Chemistry Reviews 2009; 253: 1906-1925

14. Prevost G, Marmant C, Gunning M, Thomas F. Therapeutic use and perspectives of synthetic peptides in oncology. Acta Oncol 1993 32: 209-215.

15. Hofland LJ, Visser-Wisselaar HA, Lamberts SW. Somatostatin analogs: clinical application in relation to human somatostatin receptor subtypes. J Biochem Pharm 1995; 50: 287-297.

16. Dasgupta P. Somatostatin analogues. Multiple roles in cellular proliferation, neoplacia and angiogenesis. Pharm \& Ther 2004; 102: 61-85.

17. Reubi JC, Schar JC, Waser B et al. Affinity profiles for human somatostatin receptor subtypes SST1-SST5 of somatostatin radiotracers selected for scintigraphic and radiotherapeutic use. Eur J Nucl Med 2000; 27: 273-282

18. Koumarianou E, Mikołajczak R, Pawlak D et al. Comparative study on DOTA-derivatized bombesin analog labeled with ${ }^{90} \mathrm{Y}$ and ${ }^{177} \mathrm{Lu}$ : in vitro and in vivo evaluation. Nucl Med Biol 2009; 36: 591-603.

19. Koumarianou E, Mikołajczak R, Pawlak D et al. ${ }^{44}$ Sc versus ${ }^{90} Y$ and ${ }^{177}$ Lu labelled DOTA-Bombesin and its in vitro evaluation in PC-3 cells. Eur J Nucl Med Mol Imaging 2009; 36: S309.

20. Kwekkeboom DJ, Teunissen JJ, Bakker WH et al. Radiolabeled somatostatin analog [177Lu-DOTA0,Tyr3]octreotate in patients with endocrine gastroenteropancreatic tumors. J Clin Oncol 2005; 23 2754-2762.

21. Kayani I, Bomanji JB, Groves A et al. Functional imaging of neuroendocrine tumors with combined PET/CT using ${ }^{68} \mathrm{Ga}$-DOTATATE (DOTA-DPhe1,Tyr3-octreotate) and ${ }^{18} \mathrm{~F}-\mathrm{FDG}$. Cancer 2008; 112 2447-2455. 